\title{
Multidetector CT cystography for imaging colovesical fistulas and iatrogenic bladder leaks
}

\author{
Massimo Tonolini • Roberto Bianco
}

Received: 6 July 2011 /Revised: 6 November 2011 / Accepted: 19 December 2011 / Published online: 4 February 2012

(C) European Society of Radiology 2012

\begin{abstract}
Multidetector computed tomography (MDCT) cystography currently represents the modality of choice to image the urinary bladder in traumatized patients. In this review we present our experience with MDCT cystography applications outside the trauma setting, particularly for diagnosing bladder fistulas and leaks. A detailed explanation is provided concerning exam preparation, acquisition technique, image reconstruction and interpretation. Colovesical fistulas most commonly occur as a complication of sigmoid diverticular disease, and often remain occult after extensive diagnostic work-up including cystoscopy and contrast-enhanced CT. We consistently achieved accurate preoperative visualization of colovesical fistulas using MDCT cystography. Urinary leaks and injuries represent a non-negligible occurrence after pelvic surgery, particularly obstetric and gynaecological procedures: in our experience MDCT cystography is useful to investigate iatrogenic bladder leaks or fistulas. In our opinion, MDCT cystography should be recommended as the first line modality for direct visualization or otherwise confident exclusion of both spontaneous enterovesical fistulas and bladder injuries following instrumentation procedures, obstetric or surgical interventions.

Main Messages

- Explanation of exam preparation, acquisition technique, image reconstruction and interpretation.

- Preoperative visualization of colovesical fistulas, usually secondary to sigmoid diverticulitis.

- Visualization or exclusion of iatrogenic bladder injuries following instrumentation or surgery.
\end{abstract}

M. Tonolini $(\square) \cdot$ R. Bianco

Department of Radiology, "Luigi Sacco" University Hospital,

Via G.B. Grassi 74,

20157 Milan, Italy

e-mail: mtonolini@sirm.org
Keywords Computed tomography (CT) - Urinary bladder . Urinary bladder fistula $\cdot$ Colonic diverticulitis $\cdot$ Postoperative complications

\section{Introduction}

In the past, suspected bladder injuries were investigated by means of conventional radiographic cystography, a timeconsuming and cumbersome procedure in traumatized patients that provides very limited information on surrounding pelvic structures.

During the 1990s, computed tomography (CT) cystography was developed to complement body CT in patients with possible traumatic bladder lesions. Early experiences reported this technique to be at least as accurate as radiographic studies in the assessment of bladder integrity. Besides bladder configuration and lumen, CT provides extensive information on bones, rectosigmoid, genital organs, peritoneal cul-de-sac and extraperitoneal spaces [1-5].

Recently, volumetric CT cystography acquisition on multidetector (MD) scanners benefited from improved spatial resolution and image reconstruction on arbitrary planes. Allowing identification of small urine leaks in every possible direction, MDCT cystography approaches absolute sensitivity and specificity with no false-negative results reported $[6,7]$.

Multiplanar reformations represent a highly valuable complement to axial images in providing better anatomic delineation of the rupture site, and their similar orientation to surgical anatomy is appealing to most surgeons. Therefore, MDCT cystography is currently the most recommended imaging technique to detect and classify traumatic bladder lesions, including contusion (without extravesical leak), intraperitoneal, extraperitoneal or combined rupture $[6,7]$. 


\section{MDCT cystography technique and interpretation}

Just before MDCT cystography, diluted iodinated contrast is prepared by removing $40 \mathrm{ml}$ from a $500-\mathrm{ml}$ normal saline bag and injecting a similar amount of non-ionic contrast agent (such as $350 \mathrm{mgI} / \mathrm{ml}$ iomeprol or $370 \mathrm{mgI} / \mathrm{ml}$ iopromide). Standard intravenous infusion tubing is then connected to the bag and filled with diluted contrast to avoid instilling air in the bladder.

With the patient supine on the CT scanner table, the bladder is completely emptied by placing on the floor the Foley catheter bag, which is then disposed. Preliminary unenhanced scans are optional, since recent abdominal-pelvic CT or CT urography is available for most patients.

Tubing is then connected to the Foley catheter, with the diluted contrast bag positioned 50-60 cm higher than table level. When the flow control device is opened, retrograde drip infusion of diluted contrast starts. Pressure injection and bag squeezing are strongly discouraged since bladder injury may worsen. Some serial CT scanograms may be acquired to verify progressive bladder distension and opacification. At least
$300 \mathrm{ml}$ of diluted contrast medium is injected into the bladder, unless the patient complains of intolerable bladder distension or flow stops. The estimated amount of instilled contrast mixture is recorded in the radiology report.

Then, volumetric pelvic CT is acquired at maximum bladder distension. During initial experience, acquisition parameters on our four-slice MDCT included $120 \mathrm{kV}$ tube voltage, $245-280 \mathrm{~mA}$ tube current, $4 \times 2.5 \mathrm{~mm}$ collimation, $7.5 \mathrm{~mm} /$ gantry rotation table feed (pitch 0.75 ), and $2.5 / 1.25 \mathrm{~mm}$ slice thickness/reconstruction index. Currently, acquisition on a 64-slice scanner involves tube current adjusted to body weight using automatic dose modulation, $64 \times 0.625 \mathrm{~mm}$ collimation, $0.891 \mathrm{MD}$ pitch, and $2 / 1 \mathrm{~mm}$ slice thickness/reconstruction index. Additionally, intravenous contrast medium may be used, particularly when looking for neoplastic or inflammatory processes.

Multiplanar reformations along coronal and sagittal planes are routinely performed; images are viewed at CT angiography window settings (width 600-900 HU, level 150-300 HU). Further image processing using multiplanar thick-slab sliding MIP or volume rendering
Fig. 1 A 79-year-old woman complaining of hypogastric pain, dysuria and fecaluria Cystoscopy did not identify fistulous orifices. Abdominopelvic CT showed sigmoid diverticulosis with mild signs of perivisceral and fascial inflammation, focally adherent to the thickened left bladder wall (a). Some air in the bladder lumen without previous catheterization suggested a probable colovesical fistula, which was not directly visualized during excretory phase acquisition (focused sagittal MIP image in b). At MDCT cystography, significant leakage of diluted contrast agent in the sigmoid colon was observed through a short fistula, effectively depicted (arrowheads) on axial (c) and oblique-coronal reformatted (d) images. Elective surgery included segmental resection of the sigmoid colon, resection and repair of the bladder dome
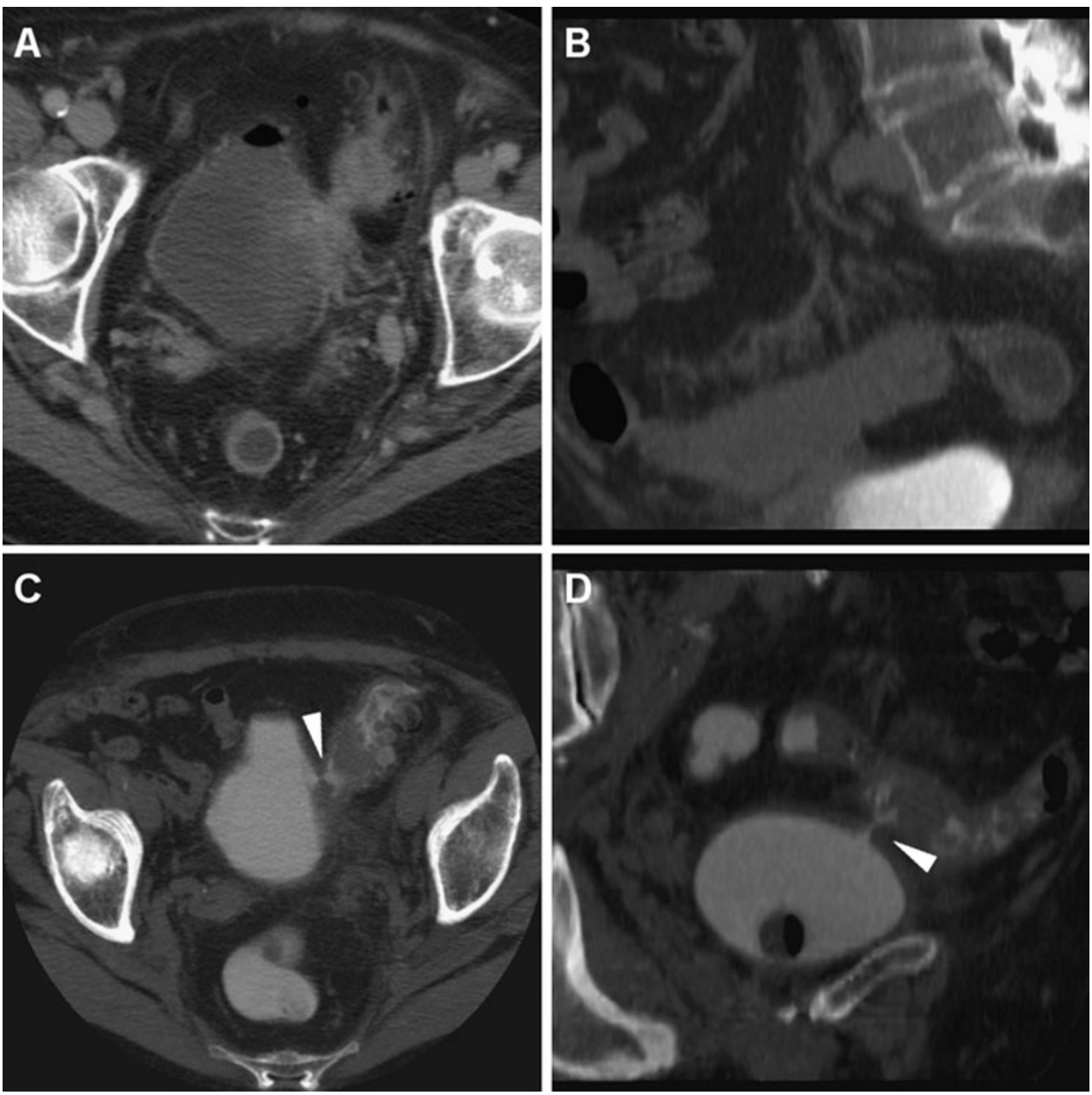
techniques helps visualization of thin fistulas or leaks (such as in Fig. 1). A normal MDCT cystogram includes a well-distended urinary bladder with thin walls, uniformly opacified (attenuation values in the range 270-320 HU) without streaky artefacts nor evidence of extraluminal contrast [4].

Borrowing from previous experiences with conventional cystography, it has been reported that bladder wall integrity may be confirmed, ruling out possible leakages, when retrograde filling with at least $250 \mathrm{ml}$ of diluted contrast is obtained. The only recognized pitfall of this technique is the possible occlusion of a small tear located at the bladder trigone by the Foley catheter balloon [6].

\section{Colovesical fistulas in diverticulitis}

Although rare, colovesical fistulas represent the most common form ( $65 \%$ of cases) of an acquired bladder-enteric fistula and most frequently occur between the recto-sigmoid and the bladder dome [8]. Diverticular disease represents their major cause (two-thirds of cases), followed by colorectal carcinoma, whereas uncommon aetiologies include Crohn's disease, bladder or uterine neoplasms, previous irradiation, female pelvic inflammatory diseases, periappendiceal abscesses penetrating trauma or foreign bodies $[9,10]$.

Clinical presentation of colovesical fistulas includes pneumaturia and fecaluria as the most pathognomonic complaints, along with variable degrees of fever, abdominal pain and weight loss $[11,12]$. Treatment includes primary surgical resection of the involved bowel segment (most usually the sigmoid) and colorectal anastomosis, plus closure of the bladder defect or vesical windowectomy; sometimes a protective colostomy is created $[8,11]$. Recently, safe and effective resection of diverticulitis complicated by enteric fistulae has been performed laparoscopically [13].

Despite an easy clinical diagnosis, precise identification of colovesical fistulas is often challenging and is obtained in only $55 \%$ of patients after extensive work-ups. Cystoscopy, conventional cystography, colonic enema, sigmoidoscopy and colonoscopy have very limited accuracy in identifying these fistulas [9-11].
Fig. 2 An 80-year-old woman undergoing routine body $\mathrm{CT}$ for long-standing, unexplained fever. Air was present in the urinary bladder, with Foley catheter balloon in place (a). MDCT cystography (axial, sagittal reformatted and oblique coronal images in $\mathbf{b}, \mathbf{c}$, and $\mathbf{d}$ respectively) obtained adequate bladder distension with instillation of $300 \mathrm{ml}$ diluted contrast medium, and visualization (arrowheads) of a subtle, initially unsuspected colovesical fistula due to colonic diverticular disease
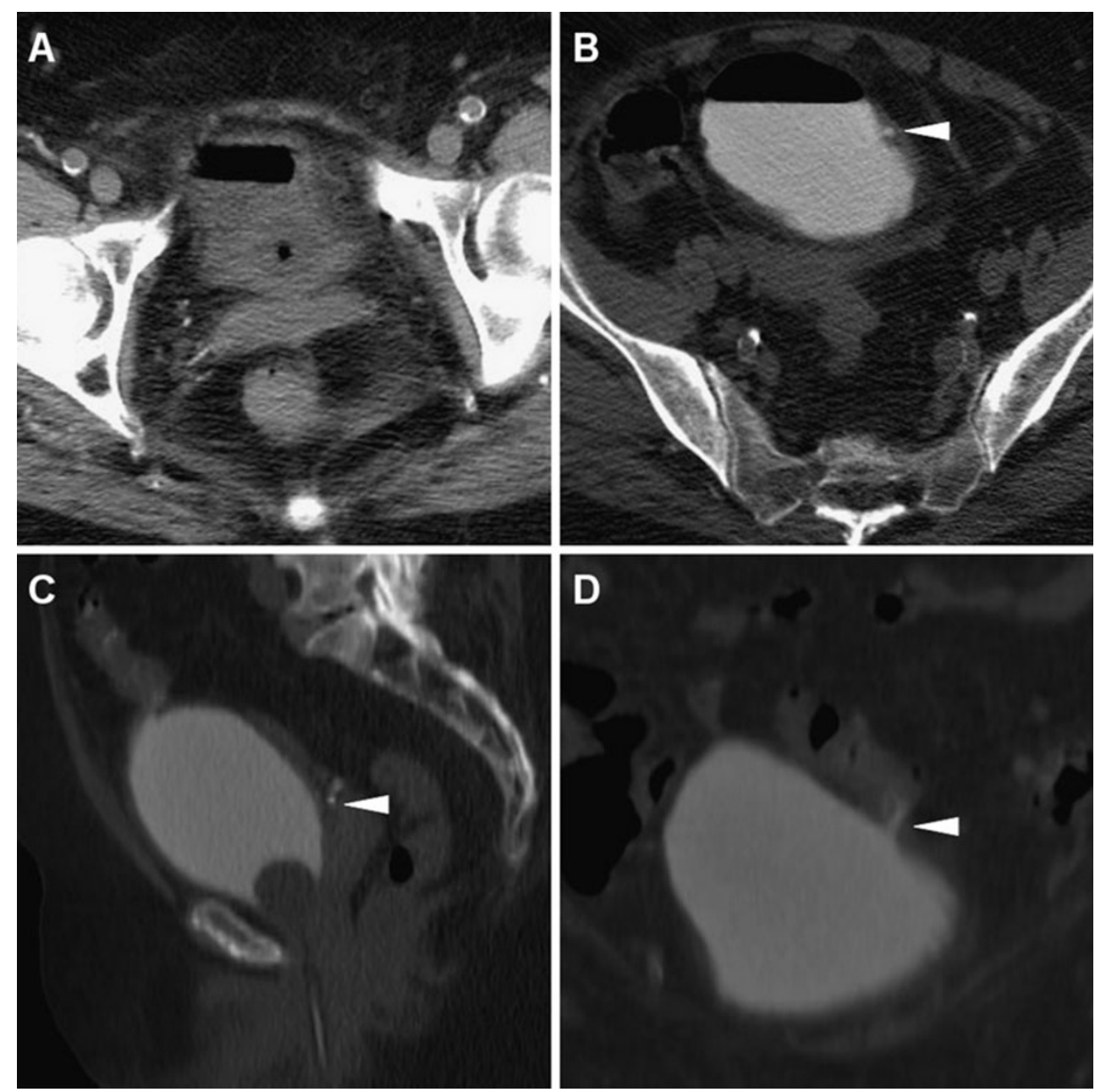
Fig. 3 A 61-year-old patient undergoing laparotomic surgery for Stage II (FIGO) bilateral ovarian cystoadenocarcinoma (preoperative staging CT image in a). MDCT cystography was performed $4 \mathrm{~h}$ after surgery because the gynaecological surgeon suspected intraoperative bladder rupture. Multiplanar assessment (axial, sagittal and coronal images in $\mathbf{a}, \mathbf{b}$ and $\mathbf{c}$, respectively) with adequate bladder filling detected some extraperitoneal air without extravasated contrast in the perivesical spaces, allowed confident exclusion of bladder rupture with urine leak and possible vesico-vaginal fistula. The patient recovered well and was discharged from hospital
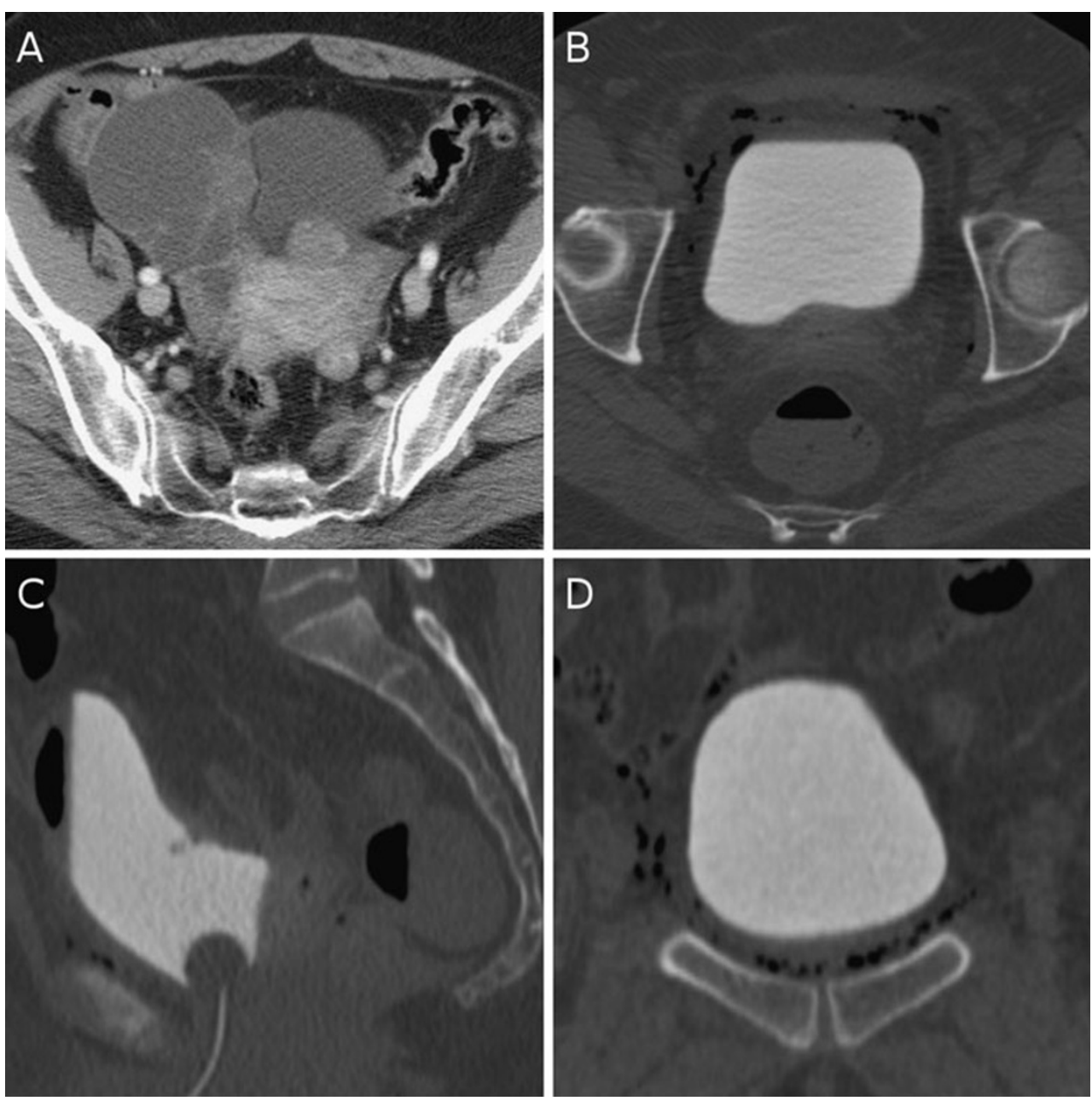

Contrast-enhanced CT with excretory phase or urographic acquisition is commonly used as the primary imaging technique to investigate suspected colovesical fistulas. Reported accuracy is variable and often limited, since tracks are directly identifiable only when filled by air, fluid or enteral contrast [10, 14]. Most commonly, fistulization is indirectly suggested by bowel-to-bladder adherence, focal bladder mural thickening and/or intraluminal air. Extraluminal CT findings such as bowel wall thickening, diverticula or an extravesical soft tissue mass corresponding to diverticulitis or carcinoma hint at the aetiology of the fistula [9, 10].

In our Department, MDCT cystography has been successfully employed to detect and visualize colovesical fistulas in patients with colonic diverticular disease. In our experience, retrograde bladder distension with at least $250 \mathrm{ml}$ diluted contrast medium achieves opening and opacification of thin yet patent colovesical fistulas. Multiplanar reformations, also using MIP algorithms, are invaluable in precisely depicting these short, small-calibre fistulous tracks, thus enabling an accurate preoperative planning (Figs. 1, 2).
Recently, sigmoid-vesical fistulas have been preoperatively investigated using MRI with high accuracy in correct identification of the underlying disease, even though the fistulous tract might not be clearly identified [12, 15]. Although magnetic resonance imaging (MRI) represents an attractive technique when radiation dose is a concern, its use in the emergency setting is largely limited by cost, availability and lengthy examination time. Our experience recommends MDCT cystography as a fast, cheaper and highly accurate technique to depict colovesical fistulization, particularly when planning for surgical or laparoscopic treatment. Also, ileovesical (most often caused by Crohn's disease) and rectovesical (associated with tumours or following trauma) fistulas can also be effectively imaged using the same modality.

\section{Iatrogenic and postoperative lesions}

Because of embryological development and anatomical proximity to the genital organs, most (61.5\%) iatrogenic 
bladder injuries occur as complications of gynaecological surgery, such as hysterectomy, pelvic masses resection, Caesarean section, diagnostic laparoscopy, anti-incontinence or laparoscopically assisted vaginal procedures [16, 17]. Predisposing factors include distortion of the pelvic anatomy by post-surgical adhesions, radiation therapy, pelvic inflammatory disease, large uterine fibroids and advanced-stage malignancies, obesity, diabetes and postoperative surgical infection [17-19].

More rarely, lower urinary tract iatrogenic lesions may occur during general surgery (particularly for colorectal cancer, diverticulitis or inflammatory bowel disease), vascular and urological interventions (such as prostatectomy and ureteral reimplantation during renal transplant) with variable reported injury rates according to their complexity [16, 17, 20, 21].

Most iatrogenic bladder lesions are full-thickness tears, commonly located at the bladder dome, and half of them are identified and treated intraoperatively. Conversely, the diagnosis of urological complications is often made postoperatively following complaints of unexplained or persistent haematuria, fever, abdominal pain and oliguria $[16,20]$.
Imaging plays a key role in the diagnosis of urological postsurgical complications and often proves invaluable to the surgeon in choosing the correct therapeutic option [17]. Since a urethral catheter is often present postoperatively, in the past conventional radiographic cystography was employed to visualize suspected bladder lacerations, with significant limitations related to obtaining an adequate distension and to superimposition of bowel and bony structures [2, 17]. Largely used to investigate postoperative pelvic abnormalities, CT can detect and classify fluid collections such as lymphocele, haematoma, abscess and urinoma with their relationship to the surrounding structures [22]. Recently, MDCT cystography has been proposed to assess the integrity of the vesicourethral anastomosis after radical prostatectomy, allowing to identify very small urine leaks [23].

With its intrinsic advantages, including speed, thin collimation and multiplanarity, MDCT cystography has in our experience proved useful to exclude (Fig. 3) or document iatrogenic bladder leakages following instrumentation procedures (Fig. 4) or surgical interventions (Fig. 5) with extremely high accuracy.
Fig. 4 An elderly, 86-year-old woman with multiple medical problems, hospitalized for right femur fracture. CT urography requested for clinical suspicion of acute pyelonephritis detected extensive presence of air in the bladder (a), but no extravesical urine leak was identified even on coronal MIP reformations (b). MDCT cystography (sagittal and coronal reformatted images in $\mathbf{c}$ and $\mathbf{d}$, respectively) clearly opacified a tear in the upper posterior aspect of the bladder (arrowheads), which was retrospectively attributed to difficult emergency catheterization manoeuvres
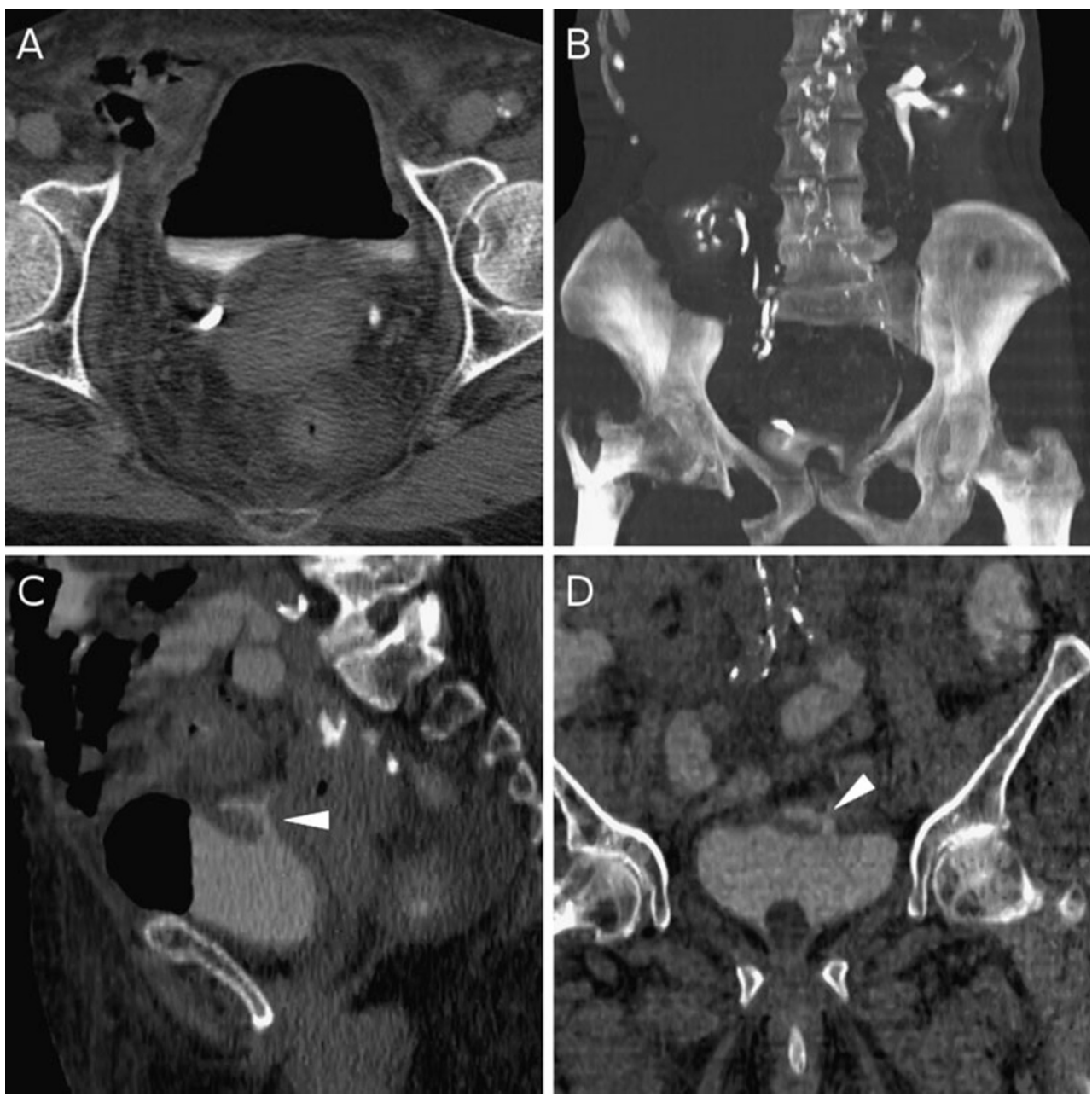
Fig. 5 A 23-year-old female investigated with conventional cystography by the attending radiologist, shortly after troublesome Caesarean section, with confirmation of bladder rupture including a fairly large right-sided pseudo-diverticular outpouching (a). MDCT cystography was requested for clarification and operative treatment planning, and revealed another large extravasation on the left upper bladder aspect (b) and fistulization from the bladder dome towards the ileum (arrowhead in c). After surgical repair, postoperative configuration of the urinary bladder was documented with radiographic cystography (d)
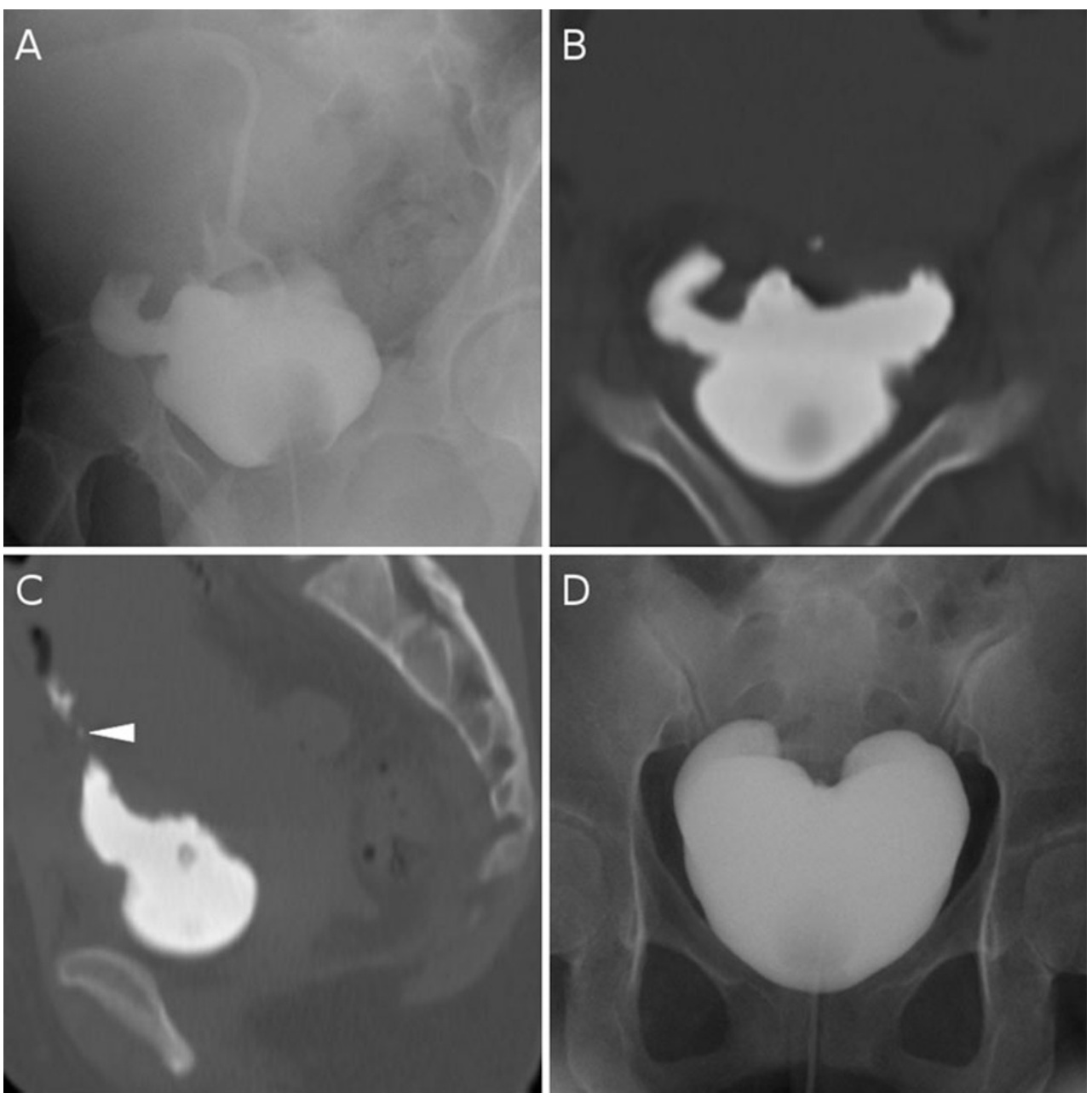

\section{Conclusion}

Based on our experience, MDCT cystography should be recommended as the most effective and accurate technique for direct visualization or exclusion of both spontaneous enterovesical fistulas and bladder leaks or fistulas following instrumentation procedures, obstetric or surgical interventions.

Acknowledgements We would like to thank the professional nurses Nerea Bevilacqua, Claudio Bonomi, Eugenia Ferron, M. Teresa Meloni and Giacomo Nocera for their valuable contribution in developing and performing the MDCT cystography technique, as well as for their daily care of our patients in the Radiology Department.

\section{References}

1. Lis LE, Cohen AJ (1990) CT cystography in the evaluation of bladder trauma. J Comput Assist Tomogr 14:386-389

2. Peng MY, Parisky YR, Cornwell EE 3rd et al (1999) CT cystography versus conventional cystography in evaluation of bladder injury. AJR Am J Roentgenol 173:1269-1272

3. Morgan DE, Nallamala LK, Kenney PJ et al (2000) CT cystography: radiographic and clinical predictors of bladder rupture. AJR Am J Roentgenol 174:89-95
4. Vaccaro JP, Brody JM (2000) CT cystography in the evaluation of major bladder trauma. Radiographics 20:1373-1381

5. Deck AJ, Shaves S, Talner L et al (2000) Computerized tomography cystography for the diagnosis of traumatic bladder rupture. $\mathrm{J}$ Urol 164:43-46

6. Chan DP, Abujudeh HH, Cushing GL Jr et al (2006) CT cystography with multiplanar reformation for suspected bladder rupture: experience in 234 cases. AJR Am J Roentgenol 187:1296-1302

7. Ishak C, Kanth N (2011) Bladder trauma: multidetector computed tomography cystography. Emerg Radiol 18:321-327

8. Carvajal Balaguera J, Camunas Segovia J, Pena Gamarra L et al (2006) Colovesical fistula complicating diverticular disease: onestage resection. Int Surg 91:17-23

9. Yu NC, Raman SS, Patel M et al (2004) Fistulas of the genitourinary tract: a radiologic review. Radiographics 24:1331-1352

10. Goldman SM, Fishman EK, Gatewood OM et al (1985) CT in the diagnosis of enterovesical fistulae. AJR Am J Roentgenol 144:1229-1233

11. Melchior S, Cudovic D, Jones J et al (2009) Diagnosis and surgical management of colovesical fistulas due to sigmoid diverticulitis. J Urol 182:978-982

12. Ravichandran S, Ahmed HU, Matanhelia SS et al (2008) Is there a role for magnetic resonance imaging in diagnosing colovesical fistulas? Urology 72:832-837

13. Nguyen SQ, Divino CM, Vine A et al (2006) Laparoscopic surgery for diverticular disease complicated by fistulae. JSLS 10:166-168

14. Werner A, Diehl SJ, Farag-Soliman M et al (2003) Multi-slice spiral CT in routine diagnosis of suspected acute left-sided colonic 
diverticulitis: a prospective study of 120 patients. Eur Radiol 13:2596-2603

15. Koketsu S, Watanabe T, Minami M et al (2009) Preoperative evaluation of enterovesical fistula using magnetic resonance imaging: case report. Hepatogastroenterology 56:696-698

16. Siow A, Nikam YA, $\mathrm{Ng} \mathrm{C}$ et al (2007) Urological complications of laparoscopic hysterectomy: a four-year review at KK Women's and Children's Hospital, Singapore. Singapore Med J 48:217-221

17. Paspulati RM, Dalal TA (2010) Imaging of complications following gynecologic surgery. Radiographics 30:625-642

18. Armenakas NA, Pareek G, Fracchia JA (2004) Iatrogenic bladder perforations: longterm followup of 65 patients. J Am Coll Surg 198:78-82
19. Ostrzenski A, Ostrzenska KM (1998) Bladder injury during laparoscopic surgery. Obstet Gynecol Surv 53:175-180

20. Gilmour DT, Dwyer PL, Carey MP (1999) Lower urinary tract injury during gynecologic surgery and its detection by intraoperative cystoscopy. Obstet Gynecol 94:883-889

21. Lind MY, Hazebroek EJ, Kirkels WJ et al (2004) Laparoscopic versus open donor nephrectomy: ureteral complications in recipients. Urology 63:36-39

22. Gayer G, Zissin R, Apter S et al (2002) Urinomas caused by ureteral injuries: CT appearance. Abdom Imaging 27:88-92

23. Lee HJ, Shin CI, Hwang SI et al (2008) MDCT cystography for detection of vesicourethral leak after prostatectomy. AJR Am J Roentgenol 191:1847-1851 